Instituto Internacional de Investigación y Desarrollo Tecnológico Educativo INDTEC, C.A.

DOI: https://doi.org/10.29394/Scientific.issn.2542-2987.2018.3.8.4.79-99

OAI-PMH: http://www.indteca.com/ojs/index.php/Revista Scientific/oai

\title{
Conocimiento Científico en la Investigación Postpositivista del Siglo XXI: De lo Externo a lo Interno del Ser
}

\author{
Autor: Erivan José Rondón Valero \\ Universidad Fermín Toro, UFT \\ erivan.rondon@gmail.com \\ Mérida, Venezuela
}

\section{Resumen}

En este nuevo siglo XXI, se están efectuando estudios postpositivistas con la finalidad de acercarse a la compresión y análisis del comportamiento dinámico del individuo dentro de las organizaciones, a través de la aplicación de métodos humanistas como holísticos, en el que los procesos intervinientes internos como externos, son vistos como un todo, asimismo con el objetivo de obtener los máximos niveles compresivos del hombre dentro de la sociedad. En este compendio analítico, así como documental, se presenta una reflexión sobre la esencia del ser, por lo que no debe seguir siendo fijado como un ser sin emociones, sentimientos, necesidades, al contrario, es importante tomar en cuenta sus elementos cualitativos, que no se pueden medir ni cuantificar desde el paradigma positivista. El comportamiento del ser humano como protagonista de todos los procesos de la vida se encuentran enmarcados en las actividades, tareas, acciones ejecutadas en la sociedad; como resultado de las relaciones humanas, profesionales, empresariales y familiares; por ello en la actualidad, representa una aproximación al cambio de paradigma investigativo del área del conocimiento científico, en el que la nueva dinámica de la globalización ha originado nuevos campos de investigación en las ciencias sociales. Finalmente, las reflexiones que se aportan.

Palabras clave: ciencia; conocimiento; investigación. 


\title{
Scientific Knowledge in the Postpositive Investigation in the 21st Century: From the External to the Inside of the Being
}

\begin{abstract}
In this new 21st century, post-positivist studies are being carried out with the purpose of approaching the compression and analysis of the dynamic behavior of the individual within organizations, through the application of humanistic and holistic methods, in which the internal intervening processes such as external, they are seen as a whole, also with the aim of obtaining the maximum levels of compression of man within society. In this analytical compendium, as well as documentary, a reflection on the essence of being is presented, so it should not remain fixed as a being without emotions, feelings, needs, on the contrary, it is important to take into account its qualitative elements, which they can not be measured or quantified from the positivist paradigm. The behavior of the human being as protagonist of all the processes of life are framed in the activities, tasks, actions executed in the society; as a result of human, professional, business and family relationships; Therefore, at present, it represents an approach to the change of the research paradigm in the area of scientific knowledge, in which the new dynamics of globalization have originated new fields of research in the social sciences. Finally, the reflections that are provided.
\end{abstract}

Keywords: science; knowledge; research. 


\section{Introducción}

En este nuevo siglo $\mathrm{XXI}$, se han emprendido mayor número de estudios cualitativos con la finalidad de acercarse a la compresión del comportamiento dinámico del individuo dentro de las organizaciones, a través de la aplicación de métodos humanistas y holísticos en el que los procesos intervinientes internos como externos son vistos como un todo; buscando obtener los máximos niveles productivos. Es importante destacar que el principal activo de cualquier tipo de organización, lo representa su personal y serán ellos sus entes innovadores y responsables de crear ventajas competitivas.

Según Kolakowski (1988): interpreta que el mundo que conocemos es un conjunto de hechos individuales observables. Nuestro saber tiende a ordenar estos hechos en un saber verdadero, esto es, algo que puede utilizarse de modo práctico y que permite prever ciertos acontecimientos en función de otros acontecimientos. En este contexto, para la humanidad la ciencia es el único medio y camino para solucionar todos los problemas individuales como sociales que abruman a los hombres. No obstante, el éxito de la ciencia consiste en su capacidad de cuantificar, explicar los hechos y fenómenos.

En este mismo orden de ideas, es importante mencionar que los aspectos cuantificables del contexto no son toda la realidad, por ello, el hombre observa y experimenta hechos de la situación, que lo conducen a la producción de nuevas ideas, dirigiéndolo a la investigación científica, por consiguiente, una opinión ampliamente compartida de la ciencia es que utiliza el método científico, como herramienta positiva para llevarlo hacia el desarrollo del conocimiento científico.

Es por lo que, durante la historia de la humanidad, se ha considerado que la ciencia constituye una parte representativa de la realidad. En su significado más general, equivale a toda clase del saber. Por tanto, la ciencia para Busot (1988): la define como "un cuerpo organizado de conocimientos 
que se revisan y renuevan continuamente para explicar la dinámica de los sucesos u objetos que se ocupa, sirviéndose para ello de una metodología finamente elaborada que responde a las exigencias de un proceso investigador crítico y rigoroso" (pág. 7). Del mismo modo, de acuerdo con el autor anteriormente mencionado, se define la ciencia como el proceso de investigación que se utiliza para explicar de forma organizada, racional, precisa y verificable, los hechos de la realidad. Es importante acotar, que la ciencia es producto de la práctica académica, organizativa y significativa de la actividad del hombre.

\section{EI Problema}

El mundo de las ciencias sociales en el siglo XXI, se encuentra relacionado al concepto de cosmovisión. Por lo tanto, para el filósofo Hume considerado en el campo de la investigación, uno de los pensadores más representativos de la tradición empirista, señala que, las ciencias guardan alguna relación con la naturaleza humana, siendo ésta, la fundamental de los saberes, en la que el hombre es el que juzga acerca de la verdad o falsedad, como centro de la sabiduría y objeto del conocimiento; plantea a la vez, que es esencial desarrollar una ciencia del hombre, que se ha de hacer aplicando la investigación empírica, como el único fundamento sólido que se logra en el saber, el cual ha de radicar en la experiencia y la observación.

En consecuencia, el surgimiento de un nuevo tipo de pensamiento es la orientación hacia un Nuevo Paradigma científico, denominado paradigma científico postpositivista (postmodernidad), representando un papel fundamental donde Martínez (2006a): sugiere que "El paradigma sistémico nace y se desarrolla a causa de las limitaciones de los procedimientos analíticos de la ciencia tradicional". (pág. 76).

De acuerdo con lo antes planteado, la capacidad de explicar la realidad y la generación del conocimiento se fundamenta en los enfoques del 
paradigma científico tradicional (positivismo) frente a nuevos paradigmas (postpositivista) y sus implicaciones para el desarrollo de la investigación; por lo tanto, se propone realizar un análisis de los mencionados paradigmas, buscando develar, como es la creación del conocimiento desde un nuevo criterio de investigación.

La propuesta comprende, la realidad del ser humano desde las estructuras cualitativas; por tal motivo se circunscribe el presente estudio a los métodos de investigación postpositivista utilizados por los profesores universitarios e investigadores del Estado Mérida, en lo que, consecuentemente se induce a reflexionar acerca de la siguiente interrogante:

¿Cómo analizan los métodos de investigación postpositivista los profesores universitarios e investigadores del Estado Mérida, en sus procesos de creación de nuevos conocimientos?

\subsection{Propósito de la Investigación}

Analizar elementos teórico - prácticos de la investigación postpositivista, utilizada por los profesores e investigadores del estado Mérida en sus procesos de creación de nuevos conocimientos.

\section{Referentes Teóricos}

\subsection{Enfoques del paradigma científico tradicional (positivismo)}

En este contexto, la orientación práctica se apoya inicialmente en el positivista Hume, rápidamente fue adoptado por Comte como su principal representante designando de manera precisa el estadio científico del saber humano. Se encuentran otros grandes empiristas como Kant, Locke, Mill y Mach, entre otros, los cuales establecen un principio de verificación, en otros términos, todo debe ser verificable en base a lo empírico, siendo válido para todas las ciencias ya sean naturales o humanas.

Por tanto, el positivismo busca sólo hechos y sus leyes, no causas ni 
principios de las esencias o sustancias, se atiene a lo positivo, a lo que está puesto o dado, renuncia a lo que es vano, intenta conocer y buscar sólo las leyes de los fenómenos, asimismo el de explicar todos los hechos mediante la aclaración material de las causas; tuvo desde un principio implicaciones políticas, religiosas, morales, igualmente las estrictamente lógicas y científicas. Se difundió y fue adoptado en Europa, América como en gran parte del mundo.

Para efectos del estudio de la corriente positivista se fundamenta en base al intelectual filósofo, matemático Comte y Hume; por su parte en lo que respecta al pensamiento de Comte (1980): establece tres estadios del conocimiento como "la ley de los tres estados" estado teológico, metafísico y científico, según él, los conocimientos pasan por tres estados teóricos distintos, tanto en el individuo como en el género humano, esta ley es el fundamento de la filosofía positivista y a su vez una teoría del conocimiento así como una filosofía de la historia.

Asimismo, Comte establece que la sociedad del futuro y el hombre racional debe fundarse sobre la ciencia, por una parte, los principios de su organización serán científicamente elaborados y por otro, es importante saber el modo científico del pensamiento, que solo puede ser explicado en la historia misma de la ciencia, esto es importante para Comte, debido que, de alguna forma quiere hacer prevalecer sus principios a partir de observaciones históricas que constituirán el modo en que se ha operado la transformación progresiva del saber humano, que tienen un carácter histórico por excelencia, pudiendo indicar su razón histórica.

En consecuencia, las inspiraciones prácticas de los hechos, tienen como principal característica, la mentalidad con orientación positivista, es decir, la realidad estudiada concerniente a la mente humana no se presta solo a la fantasía o suposición de los hechos, sino exponerse a los riesgo de la práctica, tal sería el caso del uso de las ciencias matemáticas como recurso o herramienta que fueron las primeras en tomar el camino de la fase positiva del 
desarrollo, la cual recoge en su contenido más complejo y general, las relaciones mesurables (contable) entre los fenómenos en el que interesa la cantidad, buscando el conocimiento de las leyes de la naturaleza para su dominio, por tanto, es la propiedad universal y más sencilla para todas las cosas.

\subsection{Breve reseña histórica de los positivistas}

\subsubsection{David Hume (fundador 1711-1776)}

Filósofo nacido en Edimburgo (Escocia), sus obras se destacan particularmente en el campo del conocimiento. Es considerado como uno de los pensadores más representativos de la tradición empirista. Según varios autores es considerado como el padre de la filosofía positivista, como Leszek Kolakowski en su libro titulado "La Filosofía Positivista" el cual señala al respecto que fue David Hume, una de las mentes más agudas que hayan producido los tiempos modernos y, a la vez, el verdadero padre de la filosofía positivista.

De lo antes planteado, Hume trató de fundamentar todo el peso de la filosofía en el conocimiento humano, según él es tan difícil comprender la esencia del espíritu del hombre como la esencia de los hechos naturales 0 físicos. Debido a esto, decidió aplicar a su indagación de lo humano a los métodos empíricos de la observación (dentro de este campo existe la posibilidad de formular juicios, por su puesto sin perder su carácter empírico, esto es, lo matemático el cual aportaría más acerca de la realidad de los contenidos) y el comportamiento de la especie humana, atesorando para su provecho asociaciones, así como relaciones verificadas por la experiencia.

Su filosofía investiga al hombre en sí mismo como elemento dentro del conjunto del todo, entendiéndolo siempre como una presencia aislada. Con base a Hume, señala que las ciencias guardan alguna relación con la naturaleza humana, siendo ésta la ciencia fundamental en la que el hombre es 
el que juzga acerca de la verdad o falsedad, siendo el centro de las ciencias y objeto del conocimiento.

\subsubsection{Auguste Comte (principal representante 1798-1857)}

Nació en Montpellier (Francia), es el principal representante del positivismo del siglo XIX, fue el fundador de la sociología o ciencia positiva de la sociedad, que según él debía estudiar las relaciones sociales, así como las conductas humanas como resultado de la vida colectiva. La sociología era para Comte una física de las costumbres que descubriría las leyes de las asociaciones humanas y permitiría regular el destino ético y político.

La obra de Comte es considerada como la expresión clásica de la actitud positivista, es decir, la actitud de quien afirma que tan sólo las ciencias empíricas son las adecuadas fuentes del conocimiento, para él la ciencia es reformada desde la percepción donde es un hecho sociológico y hace falta describir sus etapas pasadas, el hecho de la historia y cómo reflexionar sobre sus posibilidades futuras, en que ésta es el instrumento que sirve para ejercer las facultades humanas, siendo su propósito el de dominar las condiciones establecidas en la vida natural y social del hombre, es aparentemente, una reflexión sobre la ciencia.

No obstante, el hecho de la ciencia propone la filosofía positivista como único fundamento sólido de la vida del hombre, considerado como garantía absoluta del destino, futuro y progreso de la humanidad, en el caso del positivismo, sería la ciencia la que resultaría elevada a la categoría de infinito. En definitiva, a la reforma de la humanidad, supuso que la humanidad atraviesa en su historia, por tres estados o etapas. Comte plantea tres estados o estadios del conocimiento humano: Estado o Estadio Teológico o Religioso, Estado o Estadio Metafísico o Filosófico y Estado o Estadio Científico o Positivo. 


\subsection{Nuevo paradigma (paradigma científico postpositivista)}

El paradigma científico postpositivista, comienza a producirse hacia fines del siglo XIX y llega a su pleno desarrollo en la década de los años cincuenta y sesenta del siglo XX; contribuyendo a su progreso fundamentalmente Wittgenstein, Toulmin, Hanson, Kuhn, Feyerabend, Lakatos, Polanyi y Popper. Es por medio de éste nuevo paradigma, que se aplica el cambio total del modo de pensar y conceptualizar la realidad, el cual está adquiriendo importancia significativa en el campo de las ciencias sociales, específicamente en todo el campo de la investigación social, por lo que se constituye un medio básico de ideas, sobre la naturaleza de la realidad y de nuestros conocimientos acerca de ella, la cual forma una visión diferente del medio que nos rodea, esto quiere decir, que el postpositivismo constituye un paradigma totalmente nuevo, no sustentado en el paradigma positivista o viejo paradigma, el cual exige datos y teorías vistos de una nueva manera, los cuales adquieren un significado diferente de la realidad.

En tal sentido, el postpositivismo se fundamenta en el concepto "subjetivo", lo que significa la influencia que tienen nuestras percepciones y actitudes personales, frente a posiciones teóricas, postulados, y la tradición generalmente aceptada, reemplazando así al concepto de objetividad presente en el positivismo, afirmando que el positivismo se centra en la interpretación causal de la conducta humana, en términos de variables matemáticas o estadísticas, para Martínez (1998):

La ciencia no alberga ningún absoluto ni verdad final alguna. Tiene sus comienzos en compromisos con postulados y presupuestos, los cuales serán modificados en la medida en que nuevos hechos contradigan las consecuencias derivadas de ellos. La ciencia tendrá problemas eternos, pero no podrá dar respuestas eternas. (pág. 27).

De hecho, dentro del paradigma científico postpositivista se presentan métodos o estilos de investigación (estructuras) establecidas en la creación 
del conocimiento como lo son: Método Hermenéutico; Método Fenomenológico; Método Etnográfico; Método Naturalista; Método de Estudios endógenos; Método de Investigación-acción; Método Biográficos o de historia de vida; en ese sentido, se analizan descriptivamente.

3.3.1. Método Hermenéutico: Dilthey (citado en Martínez, 2006b):

Proceso por medio del cual conocemos la vida psíquica con la ayuda de signos sensibles que son su manifestación. Es decir que la hermenéutica tendría como misión descubrir los significados de las cosas, interpretar lo mejor posible las palabras, los escritos, los textos y los gestos, así como cualquier acto u obra, pero conservando su singularidad en el contexto del que forma parte. (pág. 119).

De acuerdo con lo presentado por el autor, es el método donde su objetivo principal es la interpretación y comprensión de los hechos y de la realidad en un momento determinado. Por tanto, la compresión no se produce por sí misma, sino la tergiversación. La hermenéutica sería el procedimiento del comprender. En consecuencia, esté método parte de que el ser humano es por naturaleza interpretativo, no existe método científico, no puede existir una sola interpretación, siempre va preexistir otras interpretaciones.

3.3.2. Método Fenomenológico: Trata de cómo el hombre percibe el mundo internamente, comprende, entiende, experimenta y vive los fenómenos de la realidad. Además, estudia casos concretos como base para el descubrimiento de lo que es esencial, generalizado y siempre comienza con la experiencia concreta, teniendo en cuenta su marco referencial. Se evidencia que es un método descriptivo, reflexivo, de exigente rigor científico, que estudia hechos, así como situaciones difíciles de observar y comunicar. 
3.3.3. Método Etnográfico: En base a Martínez (2006c):

La investigación etnográfica, en el sentido estricto, ha consistido en la producción de estudios analítico-descriptivos de las costumbres, creencias, prácticas sociales y religiosas, conocimientos y comportamiento de una cultura particular, generalmente de pueblos o tribus primitivos. La antropología cultural y social tiene en la etnografía una rama fundamental, ya que sus posiciones teóricas dependen, en último análisis, de la integridad, sensibilidad y precisión de las relaciones etnográficas. (pág. 199).

De lo anteriormente planteado, el método etnográfico se refiere a la descripción y comprensión de una unidad de cualquier grupo humano o grupo de personas acerca de su estilo de vida, costumbres, culturas y características similares, que viven juntas. No obstante, de acuerdo con Cerda (1991), afirma: que "la etnografía es el principio, un método que procura la recopilación más completa, así como exacta posible de la información necesaria para reconstruir la cultura y conocer los fenómenos sociales propios de comunidades y grupos específicos" (pág. 12). Es decir, esté tipo de investigación, es holística, naturalista, fenomenológica, hace uso de la observación y no está presente en los juicios de valor.

3.3.4. Método Naturalista: Es una combinación entre el método fenomenológico y el método etnográfico. No presenta hipótesis o problema inicial de forma explícita.

3.3.5. Método investigación endógena: Para Martínez (2006d):

La investigación endógena (investigación generada desde adentro), es una investigación etnográfica en la que los investigadores pertenecen al grupo que se investiga. Están asistidos y asesorados, no guiados por un experto externo al grupo, pero son ellos, quienes eligen el objetivo y foco de interés, escogen los procedimientos metodológicos, diseñan la investigación y la ubican dentro de su marco de referencia. (pág. 211). 
De tal manera, se trata de un tipo de investigación, aplicado en grupos humanos que comparten juntos una estructura similar de vida, que sería la unidad de análisis para el investigador o los investigadores previamente seleccionados y pertenecientes a dicho grupo, con el objetivo de estudiar sus propios problemas.

3.3.6. Método de Investigación acción: Se trata de una investigación, en el que el investigador forma parte participante del problema, en otros términos, el investigador está directamente involucrado, a través de la observación y participación directa. Uno de sus principales objetivos es conocer la forma en que las personas interpretan las estructuras sociales para desarrollar actividades comunes, a través de sus organizaciones. En la actualidad es un tipo de método utilizado en las comunidades venezolanas, por medios de sus distintas formas organizativas, por lo que se beneficia directamente a la comunidad, debido que todos están involucrados; es un proceso permanente de realimentación y cambios.

3.3.7. Investigación teórica: La investigación teórica es difícil de lograr debido que se trata de la creación de nuevas teorías que de alguna manera se han mantenido y estudiado en el tiempo, por lo cual, lo más cerca que se puede llegar, es a la aproximación de la misma.

3.3.8. Método Biográficos o de historia de vida: Define Martínez (2006e):

El sujeto es lo que se ha de conocer, pues es el único hombre que existe en la realidad concreta, y es en su historia donde se le puede captar con toda su dinámica. Además, el sujeto lleva en sí toda la realidad social vivida. En él se concreta cada grupo social al que ha pertenecido y toda la cultura en la que se ha transcurrido su existencia. Al conocer al sujeto, se conoce al grupo y la cultura tal como se dan en concreto de manera subjetiva y vivida. (pág. 260). 
Es un método utilizado para ilustrar la vida significativa de un personaje relevante para el investigador. También rechaza los paradigmas hegemónicos, reivindica la cotidianidad como respuesta a la historia oficial y el criterio de certeza reside en el sujeto o los sujetos investigados.

\section{Referente Filosófico y Epistemológico}

Es importante señalar en esta era cambiante en términos de investigación, que la teoría de las relaciones humanas es esencial para el desarrollo del comportamiento del ser humano, de acuerdo con Rodríguez (2015), expone que Elton Mayo (1880-1949), juntamente con Kart Lewin (1890-1947) y otros; justificaron la necesidad sentida en la época, de humanizar la actividad productiva del hombre, con el objetivo de alcanzar y colocar al alcance de toda la sociedad, los logros obtenidos en la investigación. En este sentido, se fundamenta que los científicos en el siglo XXI, deben fortalecer las relaciones humanas, debido que los resultados cualitativos lograran acercar a las verdaderas necesidades del hombre dentro de la sociedad, proporcionando así poder satisfacer sus necesidades individuales como colectivas.

El hombre está experimentando cambios continuos en la sociedad a la cual pertenece y forma parte, por lo tanto, la compresión de todos estos nuevos fenómenos que a lo largo de la historia de la humanidad han impactado su crecimiento y desarrollo, es fundamental para acercarse a la interpretación y análisis del por qué todos estos cambios representan factores que están marcando el desenvolvimiento del hombre en el siglo XXI. Los investigadores están obligados día a día, a acercarse al verdadero origen para bien o para mal de los fenómenos contemporáneos y buscar las posibles soluciones. 


\section{Marco Metodológico}

De acuerdo con el estudio planteado, se intenta proveer una solución aleatoria para los investigadores al momento de realizar investigaciones de tipo académicas en sus instituciones de formación, además se expone el diseño de indagación que se utiliza en el presente artículo; los participantes, el escenario y la unidad de análisis. Del mismo modo, se presentan los procedimientos e instrumentos que se utilizan para la recolección de la información.

En tal sentido la investigación está suscrita desde el paradigma cualitativo de las ciencias sociales. Martínez (2006f): con relación a lo propuesto, argumenta que la investigación cualitativa se "trata de identificar básicamente, la naturaleza profunda de las realidades, su estructura dinámica, aquella que da razón plena de su comportamiento y manifestaciones" (pág. 66). Es importante mencionar, que los informantes clave se conforman por ocho (08) investigadores ubicados en las distintas universidades y centros de investigaciones del Estado Mérida, Venezuela, los cuales presentan en su mayoría experiencia en la investigación superior a diez (10) años en sus correspondientes áreas académicas.

Por lo tanto, se atisba, que cada ser humano es distinto, desde la óptica social, emocional, cultural e ideológica. Por ello, el paradigma cualitativo aborda el análisis desde la perspectiva personal de cada uno de los informantes clave, con el objetivo de acercarse a la interpretación de la realidad. El aporte de cada individuo, es un factor determinante al momento de presentar los hallazgos significativos de la investigación. Desde esta perspectiva surgió la investigación planteada, debido a que se describió de forma detallada, las interacciones, situaciones, eventos y características particulares del grupo de participantes, actuando desde su propio marco de referencia, así como también, las actitudes que muestran los investigadores al instante de realizar sus investigaciones y los criterios personales que utilizan 
para obtener los hallazgos más significativos.

\subsection{Diseño de la Investigación}

El diseño de la investigación presente fue el de tipo de investigaciónacción. Martínez (2006g): argumenta sobre las razones del por qué este tipo de metodología representa una valiosa opción al momento de describir, debido que permite que los implicados en los procesos de investigación, actúen desde su propio contexto, adecuando los factores en beneficio de la adquisición e incorporación de nuevos conocimientos.

\subsection{Técnicas e Instrumentos de la Recolección de la Información}

No obstante, las técnicas de la recolección de la información se alcanzaron por medio de la entrevista semiestructurada, la cual permitió obtener de manera directa la percepción que tenían los investigadores en relación con el momento de realizar investigaciones científicas que contribuyan al fortalecimiento de la búsqueda del conocimiento cambiante e infinito. Asimismo, se empleó la técnica de la observación participativa, en el que para Martínez (2004): es "la técnica clásica primaria y más usada por los investigadores cualitativos para adquirir información. Para ello, el investigador vive lo más que puede con las personas o grupos que desea investigar" (pág. 89).

\section{Presentación de los Hallazgo}

En este contexto, la Triangulación de la información, se logró por medio de la invitación y aceptación previa que el investigador realizó a los informantes clave; se efectúo primeramente una reunión con cada uno de ellos, con el propósito de planificar el cronograma de visitas a la sede y la metodología de trabajo a ejecutarse. Es importante resaltar que los ocho (08) informantes de la investigación, están ubicados en los Municipios: Libertador, 
Santo Maquinas, Alberto Adriani y Rivas Dávila del Estado Mérida, los cuales forman parte de universidades y centros de investigación de la región andina (Universidad de los Andes ULA, Universidad Nacional Experimental de los Llanos Occidentales Ezequiel Zamora UNELLEZ; Universidad Politécnica Territorial de Mérida UPTM), con el objetivo de desarrollar en los investigadores seleccionados, habilidades cognitivas que le permitan fijar una postura crítica al momento de desarrollar investigaciones en sus áreas profesionales por medio de aplicación de estrategias cualitativas.

En este sentido, una de las categorías de análisis surgida, fue la habilidad pensativa de nuestro propio quehacer profesional, en el que la formación profesional, estilo de vida, experiencia y el factor ético como moral, representa una variable significativa al instante de realizar procesos investigativos dentro de cualquier paradigma: cuantitativo o cualitativo.

Por lo tanto, la aplicación de métodos como técnicas al análisis de teorías para comprender y dar lectura a la realidad, es que no existe un solo camino para crear conocimiento: se destaca más allá de la ciencia o del saber científico, como único cuerpo del discernimiento, sistemáticamente organizado, exacto y verificable, expresado numéricamente, el cual tiende a la búsqueda de objetividad denominada: presunciones, conjeturas, conjunto de leyes que están sujetas a ser verificadas o refutadas para comprender y explicar la realidad, un hecho o un fenómeno del hombre, que de alguna manera es lo que se establece como principio al positivismo. La perspectiva subjetiva y objetiva de los investigadores, representan un factor primordial en sus procesos investigativos.

Por lo cual, se reflexiona que existen otros aspectos que colman la vida del hombre, como son los factores cognitivos, emocionales y sociales: aquello que el hombre requiere y necesita durante su vida, de esta manera no se puede suponer como único objeto de conocimiento lo que se puede medir contar y verificar, el mundo de la objetividad científica es un mundo cerrado e 
inhóspito, la realidad no solo puede experimentar la razón y lo observable, comprenden a la vez aspectos cualitativos como los sentimientos, emociones, motivaciones, esto es, lo que no se puede contar o medir, la esencia natural de la existencia del hombre, es por ello, que el paradigma científico postpositivista propone una teoría del conocimiento fundamentada en las ciencias humanas.

En base a lo expuesto, los informantes clave determinaron que de acuerdo al tema a investigar, se establece el método a aplicar, pero destacando que a lo largo de su trayectoria las dos corrientes investigativas, han originado aportes reveladores a sus investigaciones. No se debe crear una independencia entre los dos paradigmas, al contrario, un proceso de integración conjunta para acercarse a la búsqueda del conocimiento cambiante e infinito, a pesar de seguir el dominio del positivismo. Se resalta que el principal activo del hombre moderno del siglo XXI, es el conocimiento.

El surgimiento de la categoría de los problemas sociales, morales, y en especial el comportamiento humano, para los informantes clave desde su experiencia investigativa, deben ser analizados desde una perspectiva científica postpositivista, que se fundamente en un sistema de ideas enteramente nuevo, basados en supuestos diferentes y contrastante de los fenómenos y hechos que rodea al hombre, los cuales permitan descubrir así como de explicar el comportamiento de las cosas en términos humanos (subjetivos), susceptibles de ser utilizados en provecho de la humanidad. Por esta razón, el fundamento cualitativo se reaparece dominante, exitoso, los cuales toman un lugar preponderante en la investigación social.

En consecuencia, el postpositivismo como nuevo paradigma de investigación, se caracteriza por ser inductivo, de realidad dinámica, con perspectiva holística, humanista, realista, adaptable a las verdaderas necesidades de la humanidad y flexibles al modo de conducir sus estudios; por lo tanto, son la nueva moda investigativa, que aportaran al desarrollo de la 
investigación del mundo actual. Sus contribuciones serán significativas para las próximas generaciones.

\section{Reflexiones del Investigador}

Desde el principio de la creación del hombre, desde la perspectiva de la teología (principio de creación) o de la ciencia (principio de evolución), se encuentran en una constante búsqueda permanente en el saber y el conocer del porqué de las cosas, motivado a ello, es donde la ciencia posiblemente toma ventaja en brindar seguridad y confianza de los fenómenos que acontecen en la realidad y en el entorno de la sociedad. Es en este cimiente, que surge la doctrina del positivismo que Comte, resume a través de su ley de los tres estados, marcando la historia del conocimiento humano.

Se concreta, que para Comte a través de la ley de los tres estados, esta es entendida como una filosofía de la historia, así como como una verdadera ciencia de la sociedad, hallada con caracteres originales y nuevos como el ser social, en definitiva, el aspecto más verdadero e interesante del positivismo, el que hace que sea realmente lo que persiguió ser para la sociedad.

No obstante, el positivismo es una línea de pensamiento y una doctrina filosófica donde se acepta como válido, el conocimiento del saber científico obtenido a través de la experimentación, con el uso del método científico, se estudian los hechos y a partir de estos, se deducen las leyes que los hacen válidos, es una doctrina normativa que está directamente vinculada con el empleo de términos tales como; saber, ciencia, conocimiento, información. Se considera que la filosofía positivista, lo que hace es basar su conocimiento en lo positivo, en lo real, dejando a un lado lo abstracto y metafísico para describir y entender el comportamiento del hombre a través de la sociedad.

En el transcurrir del tiempo se considera que el positivismo más que cualquier otro, desde sus inicios ha partido del hecho de tener la condición de adquirir conocimientos basados en todo principio de causa y efecto, 
sistemáticamente organizado, exacto, metódico, demostrable, falible de la realidad, fundamentándose en un conjunto de conocimientos verificables sobre los hechos que nos rodean, implica una relación entre la observación, descripción, experimentación, demostración y aceptación de la realidad, a través de un proceso de investigación científico para llegar a la verdad, el cual evoluciona y cambia a medida que se producen nuevas investigaciones, en definitiva, el positivismo propone el hecho de que la ciencia es el único fundamento concreto de la vida del hombre, sin tomar en cuenta sus cualidades.

El nuevo paradigma (postpositivista), nace y se desarrolla a causa de las limitaciones de los procedimientos analíticos de la ciencia tradicional, donde la nueva metodología se centra en el estudio de fenómenos y experiencias humanas, de importancia a la naturaleza socialmente construida en la realidad, la relación directa existente entre el investigador y el objeto de estudio, debido que está no busca la limitación del conocimiento por medio del simple hecho de recoger datos.

Consecuentemente, el nuevo paradigma humanista, es real, considera al ser humano como un individuo totalmente diferente el uno del otro, sin mediciones, por lo que sus aptitudes conllevan a soluciones dentro de cualquier ámbito social. Este paradigma se desarrolla dentro de un campo emocional, evolucionando al ser humano con libertad sin cercenar su creatividad científica, artística dentro del medio ambiente que lo rodea.

Es importante destacar, las diferencias que existen en los nuevos paradigmas de investigación, donde el ser humano no es tratado en mediciones estándar, por el contrario, es el creador de su propio conocimiento que a corto, mediano y largo plazo, emiten resultados que día a día le permiten vivir en una sociedad diferente y con necesidades distintas de acuerdo con la realidad de su entorno, basado a las políticas sociales, económicas y culturales en las que se desenvuelve. 


\section{Referencias}

Busot, A. (1988). Investigación Educacional. Maracaibo, Venezuela: Ediluz. Cerda, H. (1991). Los elementos de la investigación. Bogotá: El Buho.

Comte, A. (1980). Discurso Sobre el Espíritu Positivo. Madrid: Alianza Editorial, S.A.

Kolakowski, L. (1988). La Filosofía Positivista. Madrid: Ediciones Cátedra, S.A.

Martínez, M. (1998). Comportamiento Humano. Nuevos Métodos de Investigación. (2ª ed.). México: Editorial Trillas.

Martínez, M. (2006a,b,c,d,e,f,g). Comportamiento Humano. Nuevos métodos de investigación. México: Editorial Trillas.

Rodríguez, M. (2015). La gerencia interdisciplinaria lo natural y lo humano. (1… ed.). Venezuela: Ipapedi. 


\section{Erivan José Rondón Valero}

e-mail: erivan.rondon@gmail.com

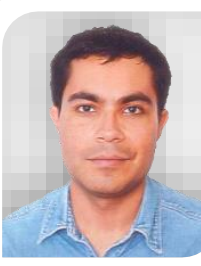

Nacido en Venezuela. Economista (Universidad de los Andes). Licenciado en Educación Mención Básica Integral. (Universidad Católica Cecilio Acosta). Postgrado en Tributos. Mención Rentas Internas (Universidad de los Andes). Componente Docente Básico en Educación Superior (Universidad de los Andes). Doctorando en Gerencia Avanzada (Universidad Fermín Toro, Extensión Mérida), Profesor Invitado Universidad UFT-Mérida en el área: Planificación Financiera y Estratégica, Profesor Invitado UNEFA-Mérida en el área: Formulación y Evaluación de Proyectos productivos, Profesional I, Departamento de Formulación y Evaluación de Proyectos PyMIS. Instituto Nacional de Desarrollo de la Pequeña y Mediana Industria INAPYMI-Mérida 2011-2017. Ponente e Investigador en diferentes áreas.

El contenido de este manuscrito se difunde bajo una Licencia de Creative Commons ReconocimientoNoComercial-Compartirlgual 4.0 Internacional 